\title{
Study of the acetylation pattern of Chitosan by pure shift NMR
}

Juan M. Lopez,* Luis F. Sánchez, Javier Nakamatsu, Helena Maruenda*

Pontificia Universidad Católica del Perú, Departamento de Ciencias - Química, CERMN, Av. Universitaria 1801, Lima, 32, Perú.

\section{SUPPORTING DATA}

\section{EXPERIMENTAL SECTION}

\section{Reagents}

Commercial chitin (C9213, batch \#061M0110V), obtained from shrimp shells (Pandalus borealis) from Iceland, was purchased from Sigma Aldrich (St. Louis, Missouri, USA). Deuterium oxide (99.98\%), $\mathrm{DCl}(30 \%)$, and $\mathrm{NaOH}$ pellets were obtained from Merck KGaA (Darmstadt, Germany). The water used was MilliQ purified (Millipore, Billerica, MA, USA). All reagents used were of analytical grade.

\section{Sample preparation:}

Chitosan samples (Chi1 - Chi20) with different degrees of acetylation ( $\mathrm{F}_{\mathrm{A}}$ from 0.001 to 0.419$)$ were prepared by alkaline deacetylation of chitin as follow:

Chi-1 - Chi-11: $3 \mathrm{~g}$ of chitin were suspended in $90 \mathrm{~mL} 50 \%(\mathrm{w} / \mathrm{w}) \mathrm{NaOH}$ solution and heated to $90^{\circ} \mathrm{C}$ with stirring at $300 \mathrm{rpm}$. A portion of the reaction mixture was removed for analysis after 9 min (Chi-1), 10 min (Chi-2), 15 min (Chi-3), 20 min (Chi-4), 25 min (Chi-5), 30 min (Chi-6), 35 min (Chi-7), 40 min (Chi-8), 60 min (Chi-9), 150 min (Chi-10), and 300 min (Chi-11). Chi12 sample was prepared under the same conditions, removed after 9 min.

Chi-13 - Chi-20: To $3 \mathrm{~g}$ of chitin, placed in a round bottom flask and left under vacuum for 24 h, $90 \mathrm{~mL}$ of a $50 \% \mathrm{NaOH}$ aqueous solution were added. The mixture was then heated to $120^{\circ} \mathrm{C}$ with stirring at $300 \mathrm{rpm}$ under nitrogen atmosphere. A portion of the reaction mixture was removed for analysis after 7 min (Chi-13), 25 min (Chi-14), 35 min (Chi-15), 40 min (Chi-16), 55 min (Chi-17), 100 min (Chi-18), 145 min (Chi-19), and 175 min (Chi-20).

The material removed in all cases was immediately washed with MilliQ water to $\mathrm{pH} 7$, dried at $50^{\circ} \mathrm{C}$ for 3 days, and kept in a closed low humidity chamber $(11 \% \mathrm{RH})$ until analysis. The NMR samples were prepared by re-dissolving $10 \mathrm{mg}$ of the recovered dried solid in $0.82 \mathrm{~mL}$ of a $0.3 \%$ (v/v) $\mathrm{DCl} / \mathrm{D}_{2} \mathrm{O}$. 


\section{NMR acquisition parameters}

${ }^{1} \mathrm{H}-\mathrm{SAPPHIRE}-\mathrm{PSYCHE}$ experiments were acquired in a pseudo 3D manner with 16 transients, $2 \mathrm{~K}$ complex data points, $5 \mathrm{KHz}$ spectral width, 8 SAPPHIRE interferogram in F2, and 32 Pure Shift interferogram with $39.063 \mathrm{~Hz}$ spectral width in F1. Total experimental time, 4 h 55 min.

${ }^{1} \mathrm{H}-{ }^{1} \mathrm{H}$-PSYCHE-TOCSY experiment was acquired with 8 transients, $2 \mathrm{~K} * 64$ complex data points, $5 \mathrm{kHz} * 4 \mathrm{kHz}$ spectral width in F2 and F1 respectively, 16 Pure Shift interferogram with 64 points per block in F3, and $80 \mathrm{~ms}$ TOCSY mixing time. Total experimental time, $12 \mathrm{~h} 52 \mathrm{~min}$. Adiabatic excitation of ${ }^{1} \mathrm{H}-\mathrm{SAPPHIRE}-\mathrm{PSYCHE}$ and ${ }^{1} \mathrm{H}-{ }^{1} \mathrm{H}-\mathrm{PSYCHE}-\mathrm{TOCSY}$ was performed with a $20^{\circ}$ flip angle double saltire CHIRP pulse, $30 \mathrm{~ms}$ duration, $10 \mathrm{KHz}$ sweep-width combined with a weak field gradient of 1.08 Gauss $/ \mathrm{cm}$.

Pure shift 2D ${ }^{1} \mathrm{H}-{ }^{13} \mathrm{C}-\mathrm{HOBS}-\mathrm{HSQC}$-TOSCY were recorded with 16 transients, $2 \mathrm{~K} * 256$ complex points, $5 \mathrm{kHz} * 20 \mathrm{kHz}$ spectral width in $\mathrm{F} 2$ and $\mathrm{F} 1$ respectively, and $80 \mathrm{~ms}$ TOCSY mixing time. Homonuclear decoupling was performed in real-time acquisition manner using HOBS scheme. We performed two $2 \mathrm{D}{ }^{1} \mathrm{H}-{ }^{13} \mathrm{C}-\mathrm{HOBS}-\mathrm{HSQC}-\mathrm{TOSCY}$ experiments: one, using 4 ms Rsnob selective inversion with $5.13 \mathrm{ppm}$ carrier position for $\mathrm{H} 1_{\mathrm{A}}$ and $\mathrm{H} 1_{\mathrm{D}}$ homonuclear decoupling and, the second one, using $6 \mathrm{~ms}$ Rsnob selective inversion with $3.59 \mathrm{ppm}$ carrier position for $\mathrm{H} 2{ }_{\mathrm{D}}$ homonuclear decoupling. Total experimental time, $13 \mathrm{~h} 42 \mathrm{~min}$.

Pure shift ${ }^{1} \mathrm{H}_{-}{ }^{13} \mathrm{C}-\mathrm{PS}-\mathrm{HSQC}$ was acquired with 16 transients, $2 \mathrm{~K} * 256$ complex points, $5 \mathrm{kHz} *$ $20 \mathrm{kHz}$ spectral width in F2 and F1 respectively. Homonuclear decoupling was performed in realtime acquisition manner using BIRD scheme. Total experimental time, $11 \mathrm{~h} 47 \mathrm{~min}$. 


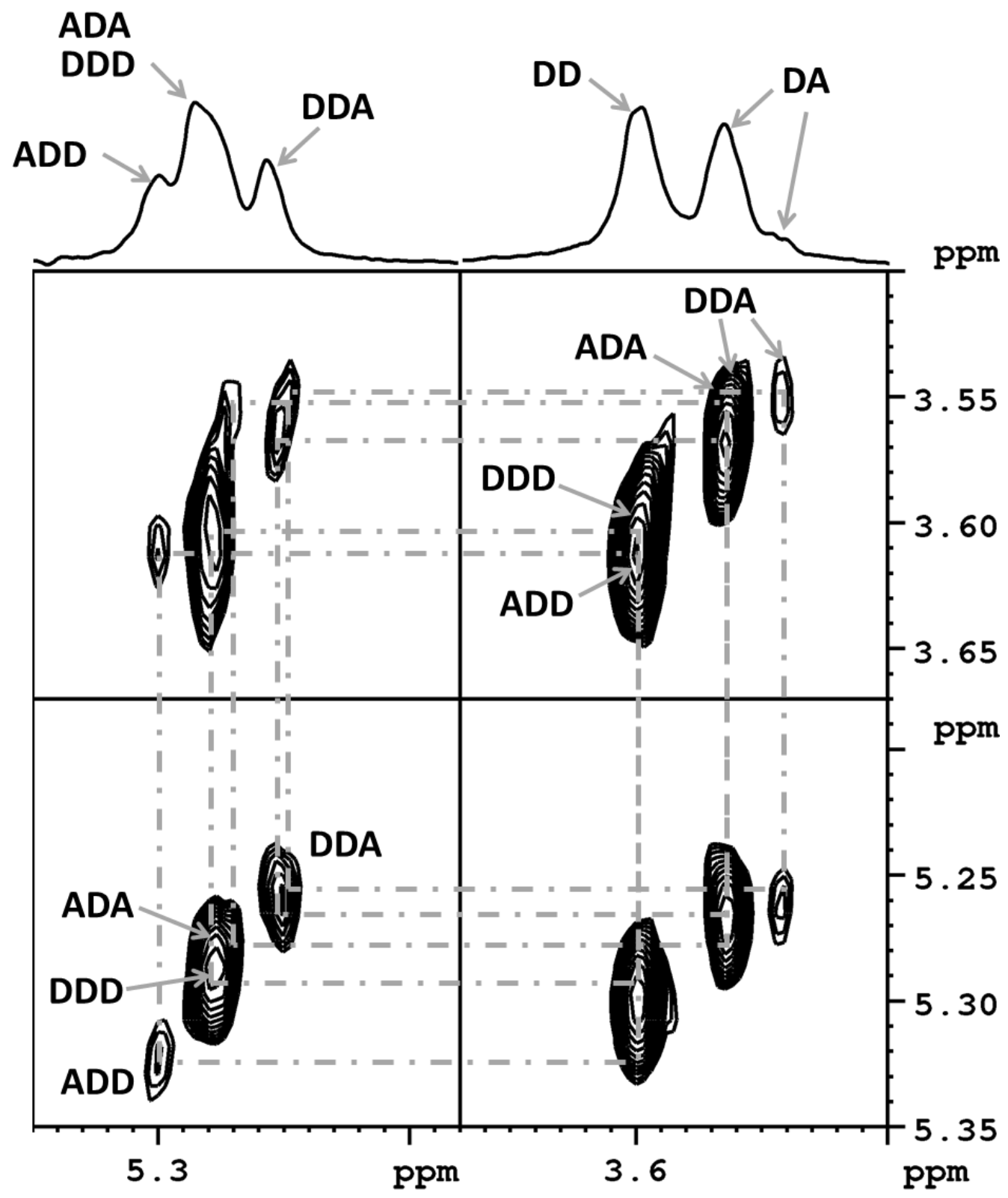

Figure S1. H1 $1_{D}$ and $H 2_{D}$ region of ${ }^{1} \mathrm{H}^{-1} \mathrm{H}-\mathrm{PSYCHE}-\mathrm{TOCSY}$ spectrum (sample Chi-1). The correlation between $\mathrm{H} 1_{\mathrm{DDA}}$ resonance $(5.26 \mathrm{ppm})$ and $\mathrm{H} 2{ }_{\mathrm{D}}$ resonance (small signal at $3.54 \mathrm{ppm}$ ) shows that it is related to DDA triads. 


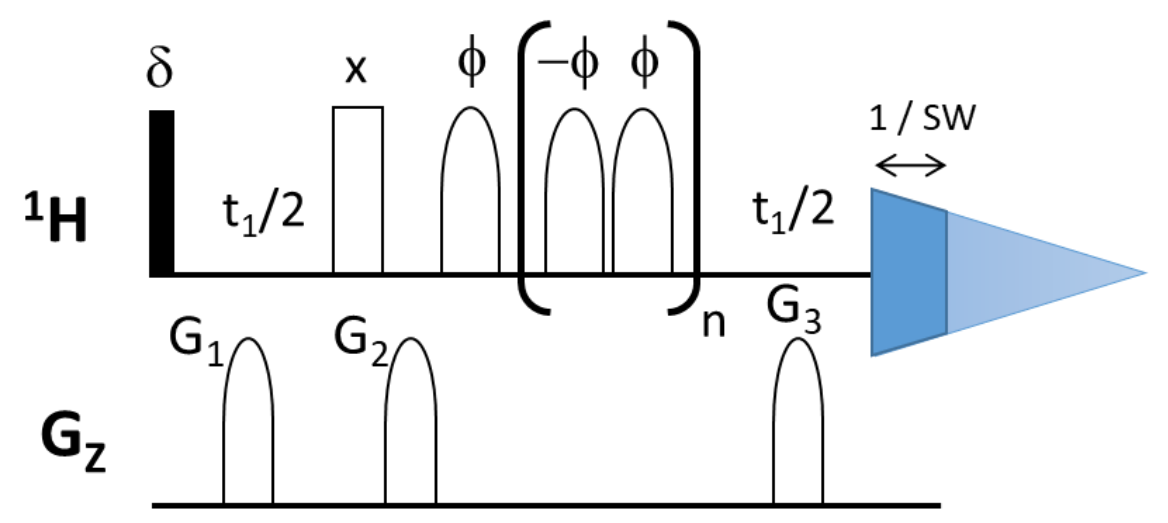

Figure S2. Modified interferogram ${ }^{1} \mathrm{H}-\mathrm{HOBS}$ pulse sequence used to measure relaxation during the selective pulse. Filled and empty rectangles indicate $90^{\circ}$ and $180^{\circ}$ pulses, respectively. Semiellipses indicate a $180^{\circ}$ selective pulse. Pulses inside the brackets are repeated $n$ times. Pulsed field gradients $G_{1}, G_{2}$, and $G_{3}$ are applied along the z-axis $\left(G_{z}\right)$. The $G_{1}, G_{2}$, and $G_{3}$ relative strength ratio were set according to $\mathrm{G}_{3}=\mathrm{G}_{1}-\mathrm{G}_{2}$. Phase cycling: $\delta=\mathrm{x}, \mathrm{x},-\mathrm{x},-\mathrm{x} ; \phi=\mathrm{x}, \mathrm{y}, \mathrm{x}, \mathrm{y}$; $\phi \mathrm{rec}=\mathrm{x},-\mathrm{x},-\mathrm{x}, \mathrm{x}$. Interferogram acquisition was achieved by acquiring part of the FID with duration $1 / \mathrm{SW}$ for each $\mathrm{t} 1$ increment.
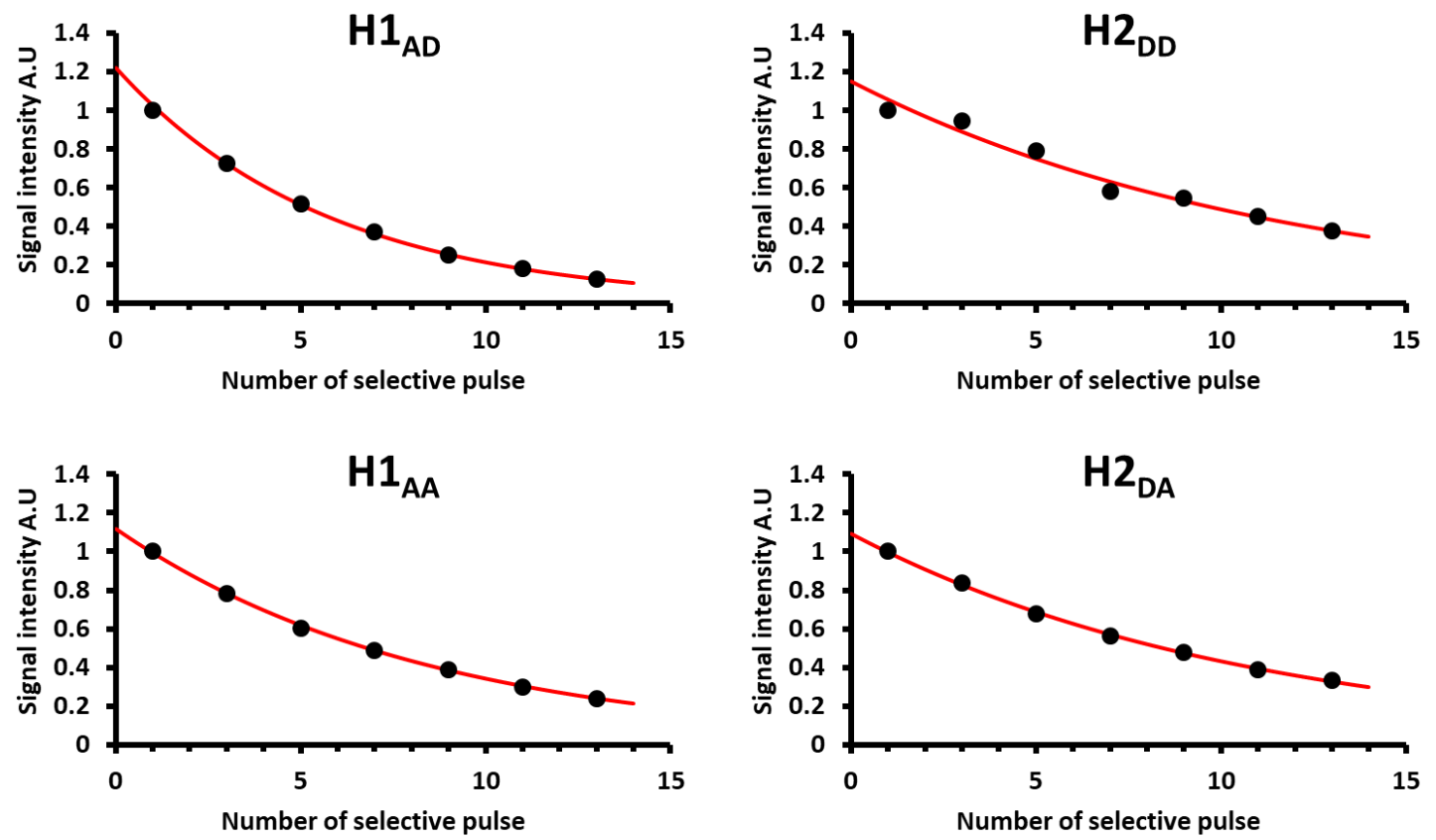

Figure S3. Relaxation during the selective pulse measured in sample Chi-3 using the pulse sequence described in Supporting Figure 2. The data was fitted according to $\mathbf{I}=\mathbf{I}_{\mathbf{0}}$ * $\operatorname{Exp}\left(-\mathbf{R}_{\text {Rsnob }} *(\mathbf{2 n}+\mathbf{1})\right)$, where $2 \mathrm{n}+1=$ total number of selective pulses. Relaxation values $\left(\mathrm{R}_{\mathrm{Rsnob}}\right)$ were obtained for $\mathrm{H} 1_{\mathrm{AD}}(0.1741), \mathrm{H} 1_{\mathrm{AA}}(0.1184), \mathrm{H} 2_{\mathrm{DD}}(0.0855)$, and $\mathrm{H} 2_{\mathrm{DA}}(0.0924)$. 


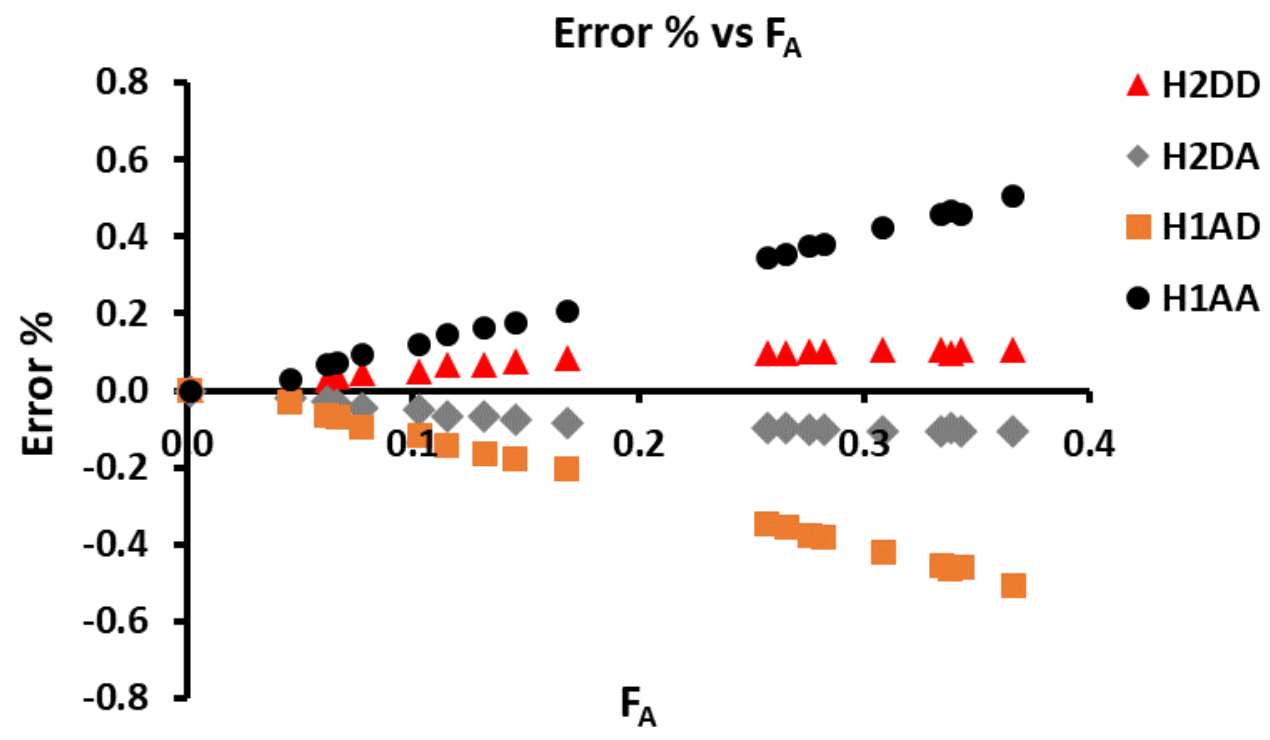

Figure S4. Error induced by the relaxation on the diads frequencies values. Experiments were recorded in interferogram manner with 8 transients, $6 \mathrm{kHz}$ spectral width, and 32 Pure Shift interferograms with 64 complex point per block and a total relaxation time of $10.34 \mathrm{~s}$. Selective inversion was achieved by a $20 \mathrm{~ms}$ Rsnob composite pulse. We performed two ${ }^{1} \mathrm{H}-\mathrm{HOBS}$ experiments with different carrier position: $4.98 \mathrm{ppm}$ for $\mathrm{H} 1_{\mathrm{A}}$ and $3.59 \mathrm{ppm}$ for $\mathrm{H} 2_{\mathrm{D}}$. The relaxation corrected diads frequencies values were calculated using the following formulas.

${ }^{1} \mathrm{H}-\mathrm{HOBS}$ experiment with 4.98 ppm carrier position:

$$
\begin{aligned}
& \mathbf{F}_{\mathbf{A D}}=\left(\mathbf{F}_{\mathbf{A}} * \mathbf{I}_{\mathbf{1 A D}} * \mathbf{C}_{\mathbf{1 A D}}\right) /\left(\mathbf{I}_{\mathbf{1 A D}} * \mathbf{C}_{\mathbf{1 A D}}+\mathbf{I}_{\mathbf{1 A A}} * \mathbf{C}_{\mathbf{1 A A}}\right) \\
& \mathbf{F}_{\mathbf{A A}}=\left(\mathbf{F}_{\mathbf{A}} * \mathbf{I}_{\mathbf{1 A A}} * \mathbf{C}_{\mathbf{1 A A}}\right) /\left(\mathbf{I}_{\mathbf{1 A D}} * \mathbf{C}_{\mathbf{1 A D}}+\mathbf{I}_{\mathbf{1 A A}} * \mathbf{C}_{\mathbf{1 A A}}\right)
\end{aligned}
$$

${ }^{1} \mathrm{H}$-HOBS experiment with 3.59 ppm carrier position:

$$
\begin{aligned}
& \mathbf{F}_{\mathbf{D A}}=\left(\left(\mathbf{1}-\mathbf{F}_{\mathbf{A}}\right) * \mathbf{I}_{\mathbf{2 D A}} * \mathbf{C}_{\mathbf{2 D A}}\right) /\left(\mathbf{I}_{\mathbf{2 D D}} * \mathbf{C}_{\mathbf{2 D D}}+\mathbf{I}_{\mathbf{2 D A}} * \mathbf{C}_{\mathbf{2 D A}}\right) \\
& \mathbf{F}_{\mathbf{D D}}=\left(\left(\mathbf{1}-\mathbf{F}_{\mathbf{A}}\right) * \mathbf{I}_{\mathbf{2 D D}} * \mathbf{C}_{\mathbf{2 D D}}\right) /\left(\mathbf{I}_{\mathbf{2 D D}} * \mathbf{C}_{\mathbf{2 D D}}+\mathbf{I}_{\mathbf{2 D A}} * \mathbf{C}_{\mathbf{2 D A}}\right)
\end{aligned}
$$

Spectra deconvolution were performed using Topspin software. Correction factors $\mathrm{C}$ were calculated by: $\mathbf{C}=\boldsymbol{e}^{-\mathbf{R}_{\mathrm{Rsnob}}}$. The figure shows a strong dependency between the error and the $\mathrm{F}_{\mathrm{A}}$ values. The error in all cases was less than $1 \%$. 

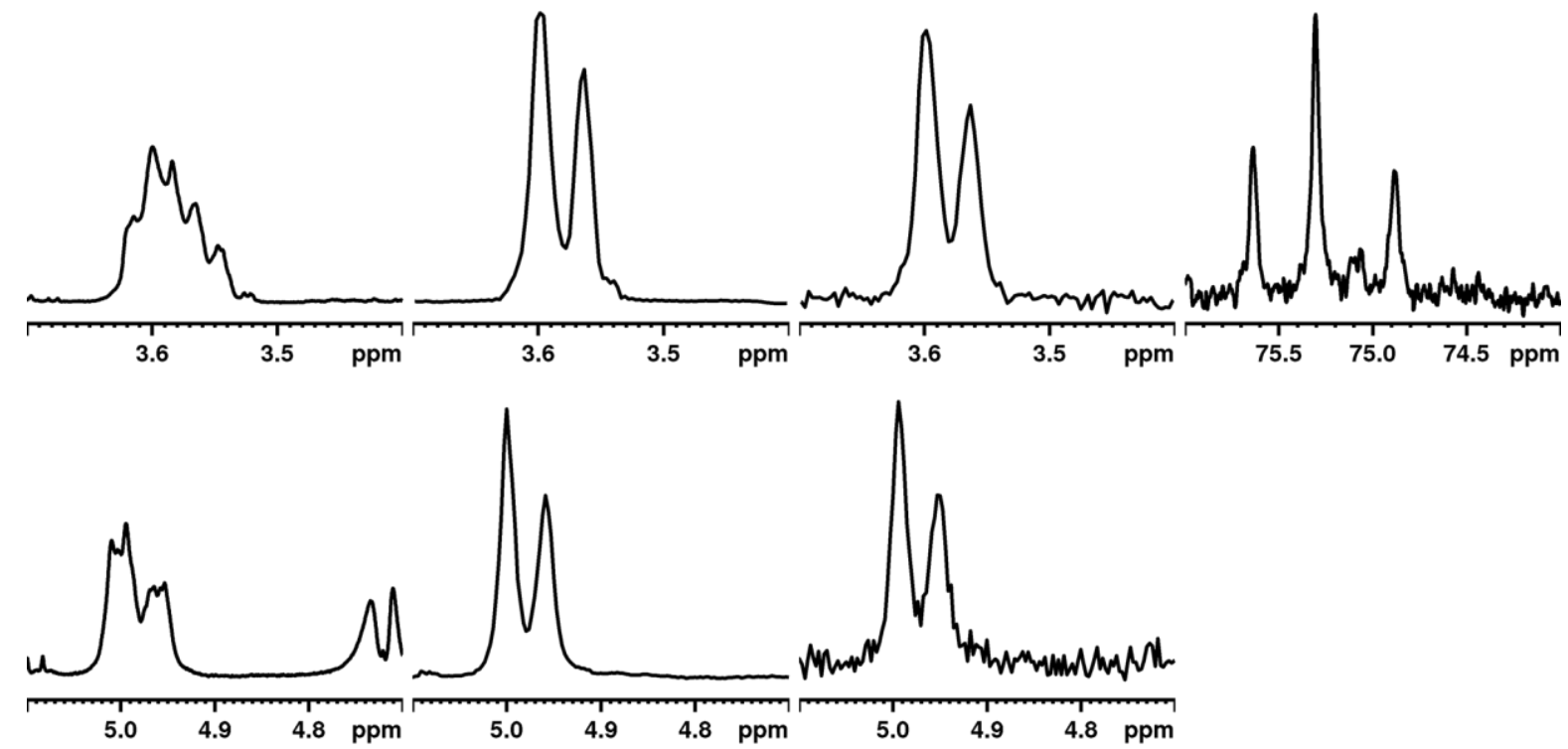

Figure S5. ${ }^{1} \mathrm{H}-\mathrm{NMR},{ }^{1} \mathrm{H}$-iHOBS and ${ }^{13} \mathrm{C}-\mathrm{NMR}$ spectra sensitivity comparison acquired on Chi12 sample. ${ }^{1} \mathrm{H}-\mathrm{NMR}$ on $12.2 \mathrm{mg} / \mathrm{mL}$ sample: $\mathrm{H} 1_{\mathrm{D}}$ (top left) and $\mathrm{H} 1_{\mathrm{A}}$ (bottom left); $1 \mathrm{H}-\mathrm{iHOBS}$ on $12.2 \mathrm{mg} / \mathrm{mL}$ sample: $\mathrm{H} 1_{\mathrm{D}}$ (top middle left) and $\mathrm{H} 1_{\mathrm{A}}$ (bottom middle left); $1 \mathrm{H}$-iHOBS on 0.45 $\mathrm{mg} / \mathrm{mL}$ sample: $\mathrm{H} 1_{\mathrm{D}}$ (top middle right) and $\mathrm{H} 1_{\mathrm{A}}$ (bottom middle right) and ${ }^{13} \mathrm{C}-\mathrm{NMR}$ on 12.2 $\mathrm{mg} / \mathrm{mL}$ sample: $\mathrm{C} 5$ (top right). ${ }^{1} \mathrm{H}-\mathrm{NMR}$ and ${ }^{1} \mathrm{H}$-iHOBS experiments were acquired with $90^{\circ}$ flip angle excitation, 8 scans, total relaxation time of $10.34 \mathrm{~s}$ and 32 increments for HOBS. ${ }^{13} \mathrm{C}-$ NMR spectra was acquired using $30^{\circ}$ flip angle, 16384 scans, $1.1 \mathrm{~s}$ acquisition time, and relaxation delay of $2 \mathrm{~s}$. 


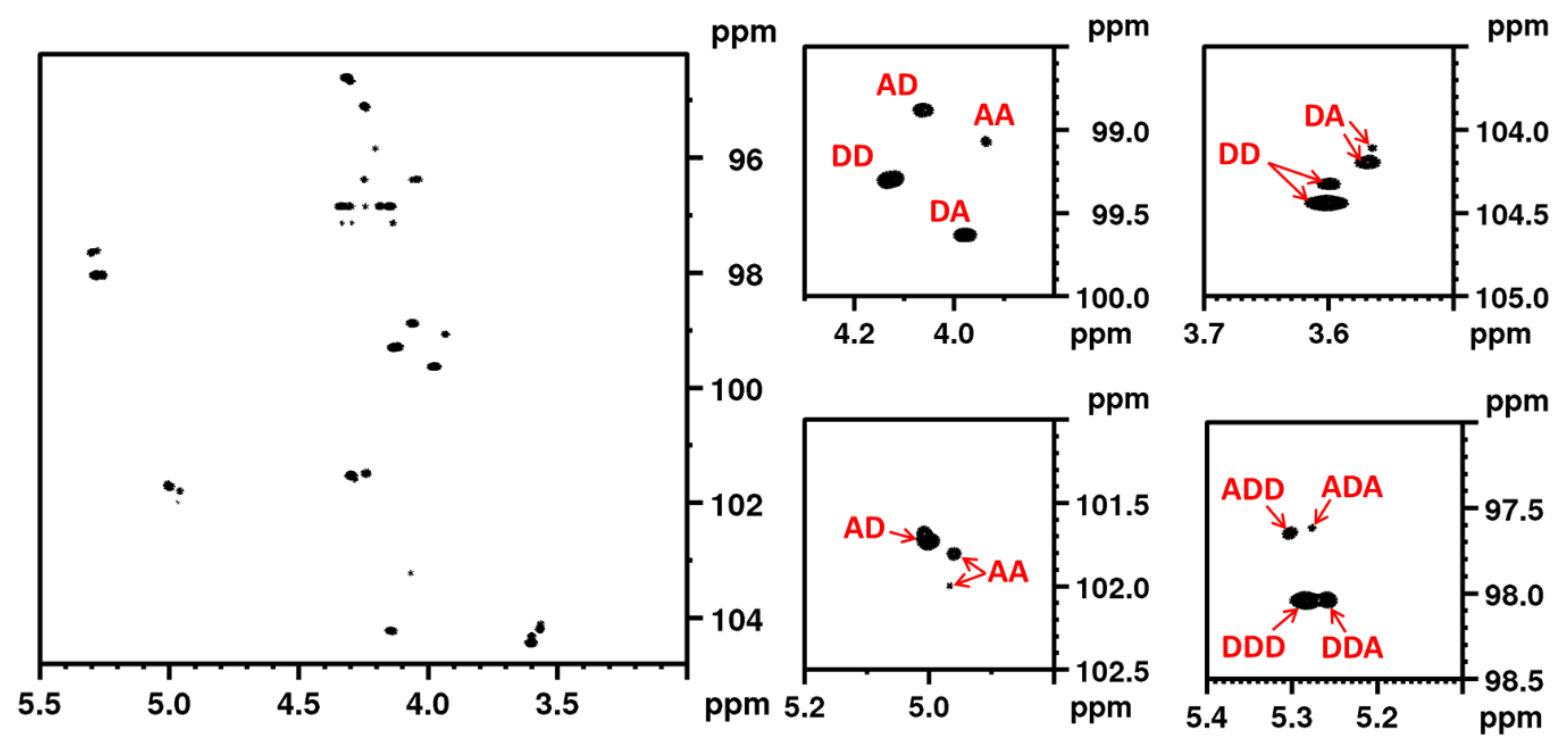

Figure S6. Showing ${ }^{1} \mathrm{H}^{13} \mathrm{C}-\mathrm{SAPS}-\mathrm{HSQC}$ (left) plus H5-C5 (top middle), deacetylated $\mathrm{H} 2-\mathrm{C} 2$ (top right), acetylated $\mathrm{H} 1-\mathrm{C} 1$ (bottom middle) and deacetylated $\mathrm{H} 1-\mathrm{C} 1$ (bottom right) expansions.

The indirect dimension enhanced resolution $(11.8 \mathrm{~Hz})$ results in an excellent separation of deacetylated $\mathrm{H} 1-\mathrm{C} 1$ triads (bottom right). Likewise, a signal splitting belonging to deacetylated $\mathrm{H} 2-\mathrm{C} 2$ (top right), acetylated $\mathrm{H} 1-\mathrm{C} 1$ (bottom middle) diads is now observed. This separation is not observed in the regular ${ }^{1} \mathrm{H}-{ }^{13} \mathrm{C}$ PS-HSQC, nevertheless those better resolved signals could not be assigned to any specific triad or tetrad.

\section{SUPPORTING TABLES}

Table S1. Signal to Noise (S/N) and diads frequencies measured from supporting figure 5 spectra.

\begin{tabular}{|c|c|c|c|c|c|c|c|c|c|c|}
\hline & Conc $(\mathrm{mg} / \mathrm{mL})$ & $\mathbf{S} / \mathbf{N}_{\text {DD }}$ & $\mathbf{S} / \mathbf{N}_{\mathbf{D A}}$ & $\mathbf{S} / \mathbf{N}_{\mathrm{AD}}$ & $\mathbf{S} / \mathbf{N}_{\mathrm{AA}}$ & $\mathbf{F}_{\text {DD }}$ & $\mathbf{F}_{\text {DA }}$ & $\mathbf{F}_{\mathrm{AD}}$ & $\mathbf{F}_{\mathrm{AA}}$ & $\mathbf{P}_{\mathrm{A}}$ \\
\hline${ }^{13} \mathrm{C}-\mathrm{NMR}$ & 12.2 & 14.9 & 8.6 & 7.2 & 3.1 & 0.376 & 0.235 & 0.257 & 0.131 & 1.05 \\
\hline \multirow{2}{*}{${ }^{1} \mathrm{H}$-iHOBS } & 12.2 & 759.0 & 611.4 & 394.1 & 267.2 & 0.349 & 0.232 & 0.241 & 0.178 & 0.975 \\
\hline & 0.45 & 31.7 & 23.0 & 13.8 & 8.9 & 0.356 & 0.224 & 0.236 & 0.183 & 0.950 \\
\hline${ }^{1}$ H-NMR & 12.2 & \multicolumn{2}{|c|}{427.0} & \multicolumn{2}{|c|}{214.8} & & & & & \\
\hline
\end{tabular}


Table S2. Fraction of acetylation $\left(\mathrm{F}_{\mathrm{A}}\right)$, diads frequencies and pattern of acetylation $\left(\mathrm{P}_{\mathrm{A}}\right)$ of twenty chitosan samples (Chi-1-Chi-20) determined by standard ${ }^{1} \mathrm{H}-\mathrm{NMR},{ }^{1} \mathrm{H}-\mathrm{iHOBS}$, and ${ }^{13} \mathrm{C}-\mathrm{NMR}$ methodologies.

\begin{tabular}{|c|c|c|c|c|c|c|c|c|c|c|c|c|}
\hline & \multirow{2}{*}{$\begin{array}{c}{ }^{1} \mathbf{H}^{\mathbf{b}} \\
\mathrm{F}_{\mathrm{A}}\end{array}$} & \multirow{2}{*}{$\begin{array}{c}{ }^{13} \mathbf{C}^{\mathbf{c}} \\
F_{A}\end{array}$} & \multicolumn{4}{|c|}{${ }^{1} \mathbf{H}^{\mathbf{d}}$} & \multicolumn{4}{|c|}{${ }^{13} \mathrm{C}^{\mathrm{c}}$} & \multirow{2}{*}{$\begin{array}{c}{ }^{1} \mathbf{H}^{\mathbf{d}} \\
\mathrm{P}_{\mathrm{A}}\end{array}$} & \multirow{2}{*}{$\begin{array}{c}{ }^{13} \mathrm{C}^{\mathbf{c}} \\
\mathrm{P}_{\mathrm{A}}\end{array}$} \\
\hline & & & $F_{D D}$ & $\mathrm{~F}_{\mathrm{DA}}$ & $\mathrm{F}_{\mathrm{AD}}$ & $\mathrm{F}_{\mathrm{AA}}$ & $\mathrm{F}_{\mathrm{DD}}$ & $\mathrm{F}_{\mathrm{DA}}$ & $\mathrm{F}_{\mathrm{AD}}$ & $\mathrm{F}_{\mathrm{AA}}$ & & \\
\hline \multirow{2}{*}{ Chi-12 } & \multirow{2}{*}{0.419} & 0.382 & 0.349 & 0.232 & 0.241 & 0.178 & 0.376 & 0.235 & 0.257 & 0.131 & 0.975 & 1.05 \\
\hline & & 0.369 & 0.350 & 0.234 & 0.239 & 0.177 & 0.429 & 0.202 & 0.227 & 0.142 & 0.975 & 0.935 \\
\hline \multirow{2}{*}{ Chi-1 } & \multirow{2}{*}{0.366} & 0.382 & 0.376 & 0.258 & 0.200 & 0.166 & 0.377 & 0.241 & 0.209 & 0.173 & 0.958 & 0.939 \\
\hline & & 0.355 & 0.376 & 0.258 & 0.191 & 0.175 & 0.409 & 0.236 & 0.211 & 0.144 & 0.935 & 0.962 \\
\hline \multirow{2}{*}{ Chi-2 } & \multirow{2}{*}{0.343} & 0.359 & 0.408 & 0.249 & 0.208 & 0.135 & 0.426 & 0.215 & 0.201 & 0.158 & 0.988 & 0.896 \\
\hline & & 0.344 & 0.395 & 0.262 & 0.204 & 0.139 & 0.417 & 0.239 & 0.196 & 0.149 & 0.998 & 0.936 \\
\hline \multirow{2}{*}{ Chi-13 } & \multirow{2}{*}{0.338} & 0.367 & 0.451 & 0.211 & 0.191 & 0.148 & 0.430 & 0.203 & 0.208 & 0.159 & 0.884 & 0.887 \\
\hline & & 0.321 & 0.445 & 0.217 & 0.185 & 0.153 & 0.528 & 0.152 & 0.160 & 0.160 & 0.879 & 0.721 \\
\hline \multirow{2}{*}{ Chi-3 } & \multirow{2}{*}{0.334} & 0.320 & 0.431 & 0.234 & 0.194 & 0.141 & 0.458 & 0.222 & 0.200 & 0.121 & 0.935 & 0.950 \\
\hline & & 0.352 & 0.432 & 0.234 & 0.186 & 0.149 & 0.459 & 0.190 & 0.231 & 0.121 & 0.913 & 0.950 \\
\hline \multirow{2}{*}{ Chi-4 } & \multirow{2}{*}{0.309} & 0.263 & 0.464 & 0.227 & 0.177 & 0.132 & 0.537 & 0.201 & 0.144 & 0.119 & 0.909 & 0.834 \\
\hline & & 0.281 & 0.459 & 0.233 & 0.174 & 0.134 & 0.503 & 0.216 & 0.159 & 0.122 & 0.910 & 0.877 \\
\hline \multirow{2}{*}{ Chi-5 } & \multirow{2}{*}{0.282} & 0.313 & 0.503 & 0.215 & 0.167 & 0.115 & 0.490 & 0.197 & 0.185 & 0.128 & 0.900 & 0.880 \\
\hline & & 0.337 & 0.493 & 0.225 & 0.168 & 0.115 & 0.512 & 0.151 & 0.184 & 0.153 & 0.916 & 0.769 \\
\hline \multirow{2}{*}{ Chi-6 } & \multirow{2}{*}{0.276} & 0.308 & 0.522 & 0.203 & 0.160 & 0.115 & 0.484 & 0.208 & 0.192 & 0.116 & 0.870 & 0.926 \\
\hline & & 0.292 & 0.517 & 0.207 & 0.158 & 0.118 & 0.516 & 0.192 & 0.161 & 0.131 & 0.868 & 0.828 \\
\hline & & 0.322 & 0.539 & 0.196 & 0.160 & 0.105 & 0.514 & 0.164 & 0.179 & 0.143 & 0.877 & 0.796 \\
\hline & & 0.243 & 0.526 & 0.209 & 0.152 & 0.113 & 0.576 & 0.181 & 0.144 & 0.099 & 0.871 & 0.842 \\
\hline & & 0.258 & & 0.196 & 0.154 & & 0.575 & 0.167 & & & 0.870 & 0.758 \\
\hline & & 0.242 & 0.531 & 0.212 & 0.154 & 0.103 & 0.561 & 0.197 & 0.130 & 0.111 & 0.897 & 0.822 \\
\hline & & 0.162 & 0.685 & 0.147 & 0.115 & 0.053 & 0.672 & 0.165 & 0.116 & 0.046 & 0.871 & 0.925 \\
\hline & & 0.131 & 0.654 & 0.177 & 0.121 & 0.048 & 0.718 & 0.151 & 0.087 & 0.044 & 0.944 & 0.872 \\
\hline & & 0.109 & 0.727 & 0.127 & 0.100 & 0.046 & 0.793 & 0.099 & 0.099 & 0.010 & 0.849 & 1.017 \\
\hline & & 0.145 & 0.697 & 0.158 & 0.103 & 0.043 & 0.716 & 0.139 & 0.145 & 0.000 & 0.910 & 1.165 \\
\hline & & 0.173 & 0.754 & 0.115 & 0.089 & 0.043 & 0.729 & 0.098 & 0.133 & 0.040 & 0.821 & 0.879 \\
\hline & & 0.202 & 0.730 & 0.138 & 0.093 & 0.039 & 0.677 & 0.120 & 0.168 & 0.035 & 0.884 & 0.981 \\
\hline & & 0.178 & 0.774 & 0.111 & 0.077 & 0.038 & 0.715 & 0.107 & 0.104 & 0.075 & 0.817 & 0.714 \\
\hline & & 0.247 & 0.751 & 0.133 & 0.080 & 0.035 & 0.640 & 0.112 & 0.091 & & 0.876 & 0.530 \\
\hline & & 0.115 & 0.815 & 0.082 & 0.074 & 0.029 & 0.783 & 0.102 & 0.095 & 0.020 & 0.816 & 0.943 \\
\hline & & 0.130 & 0.800 & 0.097 & 0.075 & 0.028 & 0.736 & 0.133 & 0.070 & 0.060 & 0.851 & 0.751 \\
\hline & & 0.050 & 0.853 & 0.069 & 0.054 & 0.024 & 0.918 & 0.032 & 0.034 & 0.015 & 0.787 & 0.718 \\
\hline & 0.010 & 0.109 & 0.837 & 0.085 & 0.054 & 0.023 & 0.791 & 0.101 & 0.078 & 0.031 & 0.826 & 0.845 \\
\hline & & 0.082 & 0.876 & 0.058 & 0.050 & 0.016 & 0.882 & 0.036 & 0.074 & 0.007 & 0.824 & 0.941 \\
\hline Chi-19 & & 0.135 & 0.846 & 0.087 & 0.051 & 0.016 & 0.783 & 0.082 & 0.080 & 0.055 & 0.887 & 0.689 \\
\hline & & 0.123 & 0.895 & 0.043 & 0.046 & 0.016 & 0.792 & 0.085 & 0.096 & 0.027 & 0.785 & 0.875 \\
\hline & & 0.070 & 0.865 & 0.073 & 0.045 & 0.017 & 0.875 & 0.055 & 0.045 & 0.025 & 0.837 & 0.717 \\
\hline & & 0.000 & 0.924 & 0.030 & 0.040 & 0.006 & 0.999 & 0.001 & 0.000 & 0.000 & 0.890 & 0.554 \\
\hline & $0.04 \mathrm{C}$ & $\mathrm{nd}^{\mathrm{e}}$ & 0.856 & 0.099 & 0.034 & 0.011 & $\mathrm{nd}^{\mathrm{e}}$ & $\mathrm{nd}^{\mathrm{e}}$ & $\mathrm{nd}^{\mathrm{e}}$ & $\mathrm{nd}^{\mathrm{e}}$ & 0.930 & $\mathrm{nd}^{\mathrm{e}}$ \\
\hline & & 0.000 & 0.998 & 0.000 & 0.001 & 0.000 & 1.000 & 0.000 & 0.000 & 0.000 & 1.001 & 1.000 \\
\hline & & $\mathrm{nd}^{\mathrm{e}}$ & 0.999 & 0.000 & 0.001 & 0.000 & $n \mathrm{~d}^{\mathrm{e}}$ & $\mathrm{nd}^{\mathrm{e}}$ & $\mathrm{nd}^{\mathrm{e}}$ & $\mathrm{nd}^{\mathrm{e}}$ & 1.001 & $\mathrm{nd}^{\mathrm{e}}$ \\
\hline
\end{tabular}

${ }^{a}$ Chi sample data was deconvoluted using Topspin software (first row on each) and Mestrenova (second row on each). ${ }^{b} \mathrm{~F}_{\mathrm{A}}$ values were calculated using classical ${ }^{1} \mathrm{H}-\mathrm{NMR}$ spectra. ${ }^{14,15} \mathrm{c}$ Inverse gate ${ }^{13} \mathrm{C}$-NMR spectra ${ }^{10}$ were used for $\mathrm{F}_{\mathrm{A}}$ and $\mathrm{P}_{\mathrm{A}}$ determinations. ${ }^{\mathrm{d}}{ }^{1} \mathrm{HOBS}$ methodology implemented in this study was used to calculate diads fractions and $\mathrm{P}_{\mathrm{A}}$ values. ${ }^{\mathrm{e}}$ nd cannot be determined. 
Table S3. Fraction of acetylation and diads frequencies of chitosan samples (Chi3, Chi-9, Chi12 and Chi-15) determined by ${ }^{1} \mathrm{H}-\mathrm{NMR},{ }^{1} \mathrm{H}-{ }^{13} \mathrm{C}-\mathrm{SAPS}-\mathrm{HSQC},{ }^{1} \mathrm{H}-\mathrm{iHOBS}$, and ${ }^{13} \mathrm{C}-\mathrm{NMR}$ methodologies.

\begin{tabular}{|c|c|c|c|c|c|c|c|c|c|c|c|c|c|}
\hline & ${ }^{1} \mathbf{H}$ & \multicolumn{4}{|c|}{ SAPS-HSQC } & \multicolumn{4}{|c|}{${ }^{1} \mathrm{H}$-iHOBS } & \multicolumn{4}{|c|}{${ }^{13} \mathrm{C}$} \\
\hline & $\mathrm{F}_{\mathrm{A}}$ & $\mathrm{F}_{\mathrm{DD}}$ & $\mathrm{F}_{\mathrm{DA}}$ & $\mathrm{F}_{\mathrm{AD}}$ & $\mathrm{F}_{\mathrm{AA}}$ & $\mathrm{F}_{\mathrm{DD}}$ & $\mathrm{F}_{\mathrm{DA}}$ & $\mathrm{F}_{\mathrm{AD}}$ & $\mathrm{F}_{\mathrm{AA}}$ & $\mathrm{F}_{\mathrm{DD}}$ & $\mathrm{F}_{\mathrm{DA}}$ & $\mathrm{F}_{\mathrm{AD}}$ & $\mathrm{F}_{\mathrm{AA}}$ \\
\hline \multirow{2}{*}{ Chi-12 } & \multirow{2}{*}{0.419} & $0.385^{a}$ & $0.211^{\mathrm{a}}$ & $0.261^{\mathrm{a}}$ & $0.143^{\mathrm{a}}$ & \multirow{2}{*}{0.349} & \multirow{2}{*}{0.232} & \multirow{2}{*}{0.241} & \multirow{2}{*}{0.178} & \multirow{2}{*}{0.376} & \multirow{2}{*}{0.235} & \multirow{2}{*}{0.257} & \multirow{2}{*}{0.131} \\
\hline & & $0.361^{\mathrm{b}}$ & $0.219^{b}$ & $0.245^{\mathrm{b}}$ & $0.175^{\mathrm{b}}$ & & & & & & & & \\
\hline \multirow{2}{*}{ Chi-3 } & \multirow{2}{*}{0.334} & $0.526^{\mathrm{a}}$ & $0.172^{\mathrm{a}}$ & $0.216^{\mathrm{a}}$ & $0.086^{\mathrm{a}}$ & \multirow{2}{*}{0.431} & \multirow{2}{*}{0.234} & \multirow{2}{*}{0.194} & \multirow{2}{*}{0.141} & \multirow{2}{*}{0.458} & \multirow{2}{*}{0.222} & \multirow{2}{*}{0.200} & \multirow{2}{*}{0.121} \\
\hline & & $0.493^{\mathrm{b}}$ & $0.246^{\mathrm{b}}$ & $0.188^{b}$ & $0.073^{\mathrm{b}}$ & & & & & & & & \\
\hline \multirow{2}{*}{ Chi-9 } & \multirow{2}{*}{0.168} & $0.717^{\mathrm{a}}$ & $0.112^{\mathrm{a}}$ & $0.107^{a}$ & $0.064^{\mathrm{a}}$ & \multirow{2}{*}{0.685} & \multirow{2}{*}{0.147} & \multirow{2}{*}{0.115} & \multirow{2}{*}{0.053} & \multirow{2}{*}{0.672} & \multirow{2}{*}{0.165} & \multirow{2}{*}{0.116} & \multirow{2}{*}{0.046} \\
\hline & & $0.676^{\mathrm{b}}$ & $0.132^{b}$ & $0.122^{b}$ & $0.070^{\mathrm{b}}$ & & & & & & & & \\
\hline \multirow{2}{*}{ Chi-15 } & \multirow{2}{*}{0.132} & $0.796^{\mathrm{a}}$ & $0.071^{\mathrm{a}}$ & $0.081^{\mathrm{a}}$ & $0.030^{\mathrm{a}}$ & \multirow{2}{*}{0.754} & \multirow{2}{*}{0.115} & 0080 & $0 \Omega 43$ & 0770 & 0098 & 0132 & 040 \\
\hline & & $0.770^{\mathrm{b}}$ & $0.099^{b}$ & $0.079^{b}$ & $0.052^{b}$ & & & 0.089 & 0.043 & 0.129 & 0.090 & 0.153 & 0.040 \\
\hline
\end{tabular}

${ }^{1} \mathrm{H}-{ }^{13} \mathrm{C}-\mathrm{SAPS}-\mathrm{HSQC}$ quantification is obtained by direct integration of the signal H5-C5 or by combining integrations from acetylated $\mathrm{H} 1-\mathrm{C} 1$ and deacetylated $\mathrm{H} 2-\mathrm{C} 2$ signals.

${ }^{\text {a }}$ Diads frequencies determined ${ }^{1} \mathrm{H}-{ }^{13} \mathrm{C}-\mathrm{SAPS}-\mathrm{HSQC}$ by integrating H5-C5 signals:

$$
\begin{aligned}
& \mathbf{F}_{\mathbf{D A}}=\mathbf{I}_{\mathbf{5 D A}} /\left(\mathbf{I}_{\mathbf{5 D A}}+\mathbf{I}_{\mathbf{5 A D}}+\mathbf{I}_{\mathbf{5 D D}}+\mathbf{I}_{\mathbf{5 A A}}\right) \\
& \mathbf{F}_{\mathbf{A D}}=\mathbf{I}_{\mathbf{5 A D}} /\left(\mathbf{I}_{\mathbf{5 D A}}+\mathbf{I}_{\mathbf{5 A D}}+\mathbf{I}_{\mathbf{5 D D}}+\mathbf{I}_{\mathbf{5 A A}}\right) \\
& \mathbf{F}_{\mathbf{D D}}=\mathbf{I}_{\mathbf{5 D D}} /\left(\mathbf{I}_{\mathbf{5 D A}}+\mathbf{I}_{\mathbf{5 A D}}+\mathbf{I}_{\mathbf{5 D D}}+\mathbf{I}_{\mathbf{5 A A}}\right) \\
& \mathbf{F}_{\mathbf{A A}}=\mathbf{I}_{\mathbf{5 A A}} /\left(\mathbf{I}_{\mathbf{5 D A}}+\mathbf{I}_{\mathbf{5 A D}}+\mathbf{I}_{\mathbf{5 D D}}+\mathbf{I}_{\mathbf{5 A A}}\right)
\end{aligned}
$$

${ }^{b}$ Diads frequencies determined ${ }^{1} \mathrm{H}-{ }^{13} \mathrm{C}$-SAPS-HSQC by integrating deacetylated $\mathrm{H} 2-\mathrm{C} 2$ and acetylated H1-C1 signals:

$$
\begin{gathered}
\mathbf{F}_{\mathbf{D A}}=\left(\left(\mathbf{1}-\mathbf{F}_{\mathbf{A}}\right) * \mathbf{I}_{2 \mathrm{DA}}\right) /\left(\mathbf{I}_{\mathbf{2 D D}}+\mathbf{I}_{\mathbf{2 D A}}\right) \\
\mathbf{F}_{\mathbf{D D}}=\left(\left(\mathbf{1}-\mathbf{F}_{\mathbf{A}}\right) * \mathbf{I}_{\mathbf{2 D D}}\right) /\left(\mathbf{I}_{\mathbf{2 D D}}+\mathbf{I}_{\mathbf{2 D A}}\right) \\
\mathbf{F}_{\mathbf{A D}}=\left(\mathbf{F}_{\mathbf{A}} * \mathbf{I}_{\mathbf{1 A D}}\right) /\left(\mathbf{I}_{\mathbf{1 A D}}+\mathbf{I}_{\mathbf{1 A A}}\right) \\
\mathbf{F}_{\mathbf{A A}}=\left(\mathbf{F}_{\mathbf{A}} * \mathbf{I}_{\mathbf{1 A A}}\right) /\left(\mathbf{I}_{\mathbf{1 A D}}+\mathbf{I}_{\mathbf{1 A A}}\right)
\end{gathered}
$$


;Selective pulse Relaxation reset_HOBS_1d-R

;avance-version (14/08/29)

;pseudo 2D sequence

; band selective homodecoupling using a HOBS element

;

;

; $\$$ CLASS=HighRes

;\$IM=2D

;\$TYPE=

;\$SUBTYPE=

;COMMENT=

\#include $<$ Avance.incl $>$

\#include $<$ Grad.incl $>$

\#include $<$ Delay.incl $>$

\author{
"d11=30m" \\ "d12=20u" \\ "in $0=\mathrm{dw}^{*} 131 "$ \\ "d0=3u"
}

"p2=2*p1"

"DELTA2 $=131 * 2 * d w "$ 
"DELTA3 $=\mathrm{p} 16+\mathrm{d} 16+\mathrm{d} 0 * 2+\mathrm{de}+\mathrm{dw}^{*} 2 * 130+5 \mathrm{u} "$

$" 129=2 * 131 * \operatorname{td} 1 "$

$1 \mathrm{ze}$

$2 \mathrm{~d} 11$

$3 \mathrm{~d} 12$

d1 pl1:f1

50u UNBLKGRAD

(p1 ph3):f1

d0

p16:gp1*0.5

d16

(p2 ph5):f1

p16:gp $1 *-0.5$

d16

DELTA3

(p47:sp34 ph2):f1

4 (p47:sp34 ph12):f1

(p47:sp34 ph2):f1

lo to 4 times 111 
$\mathrm{p} 16: \mathrm{gp} 1 *-1.0$

$\mathrm{d} 16$

$\mathrm{d} 0$

4u BLKGRAD

go $=2 \mathrm{ph} 31$

$\mathrm{d} 11 \mathrm{mc} \# 0$ to 2

F1QF(caldel $(\mathrm{d} 0,+$ in 0$))$

\section{DELTA2}

exit

$\mathrm{ph} 2=0 \quad 1$

ph3=0 022

$\mathrm{ph} 4=0$

$\mathrm{ph} 5=0$

ph12=2 3

ph31=0 220

;p11 : fl channel - power level for pulse (default)

;sp30: f1 channel - shaped pulse 180 degree (Bip720,50,20.1)

;sp34: f1 channel - shaped pulse 180 degree for Zangger-Sterk element

;p1 : f1 channel - 90 degree high power pulse

;p16: homospoil/gradient pulse

;p44: f1 channel - 180 degree shaped pulse for refocussing 
;d0 : incremented delay (2D)

[3 usec]

;d1 : relaxation delay; $1-5 * \mathrm{~T} 1$

;d11: delay for disk $\mathrm{I} / \mathrm{O}$

[30 msec]

;d12: delay for power switching

[20 usec]

;d16: delay for homospoil/gradient recovery

;11: n 180 degree shaped pulse

;129: total number of points in reconstructed FID

;130: number of complex points at the beginning not to be included

; in reconstruction

;131: number of complex points along the acquisition dimension per block

; $\quad$ block length about 8 to $10 \mathrm{~ms}$

;in $0:=\mathrm{dw}^{*} 131$

;ns: $1 *$ n, total number of scans: NS * TD0

;ds: 4

;FnMODE: QF

;for z-only gradients:

;gpz0: $2 \%$

;gpz1: 19\%

;use gradient files:

;gpnam0: RECT.1

;gpnam1: SMSQ10.100

;use AU-program proc_reset to process data 